DESY 02-001

hep-ph/0201139

\title{
Black Holes at Neutrino Telescopes
}

\author{
M. Kowalski \\ Deutsches Elektronen-Synchrotron DESY, Zeuthen, Germany \\ A. Ringwald, H. Tu \\ Deutsches Elektronen-Synchrotron DESY, Hamburg, Germany
}

\begin{abstract}
In scenarios with extra dimensions and $\mathrm{TeV}$-scale quantum gravity, black holes are expected to be produced in the collision of light particles at center-of-mass energies above the fundamental Planck scale with small impact parameters. Black hole production and evaporation may thus be studied in detail at the Large Hadron Collider (LHC). But even before the LHC starts operating, neutrino telescopes such as AMANDA/IceCube, ANTARES, Baikal, and RICE have an opportunity to search for black hole signatures. Black hole production in the scattering of ultrahigh energy cosmic neutrinos on nucleons in the ice or water may initiate cascades and through-going muons with distinct characteristics above the Standard Model rate. In this Letter, we investigate the sensitivity of neutrino telescopes to black hole production and compare it to the one expected at the Pierre Auger Observatory, an air shower array currently under construction, and at the LHC. We find that, already with the currently available data, AMANDA and RICE should be able to place sensible constraints in black hole production parameter space, which are competitive with the present ones from the air shower facilities Fly's Eye and AGASA. In the optimistic case that a ultrahigh energy cosmic neutrino flux significantly higher than the one expected from cosmic ray interactions with the cosmic microwave background radiation is realized in nature, one even has discovery potential for black holes at neutrino telescopes beyond the reach of LHC.
\end{abstract}


1. The possibility that black holes will be produced in the collision of two light particles at center-of-mass $(\mathrm{cm})$ energies above the Planck scale with small impact parameters [1] seems now within reach in the context of theories with $\delta=D-4 \geq 1$ large compact [2] or warped [3] extra dimensions and a low fundamental Planck scale $M_{D} \gtrsim 1 \mathrm{TeV}$ characterizing quantum gravity. In these theories one might expect the copious production of black holes in high energy collisions at cm energies above $M_{D}$ [4. Correspondingly, the Large Hadron Collider (LHC) [7, expected to have a first physics run in 2006, may turn into a factory of black holes at which their production and evaporation may be studied in detail [8, 9, 10, 11].

Black hole production and subsequent decay in the scattering of ultrahigh energy cosmic neutrinos on nucleons in the atmosphere may initiate quasi-horizontal air showers far above the Standard Model rate [12, 13] and with distinct characteristics 14. The reach of the Pierre Auger Observatory [15] for extensive air showers, expected to be completed by the end of 2003, in the black hole production parameter range has been thoroughly investigated in Refs. [12, 16, 17. It was found that, depending on the size of the flux of ultrahigh energy cosmic neutrinos, the first signs of black hole production may be observed at Auger already before the start of the LHC. Furthermore, it was shown that already now sensible constraints on black hole production can be obtained from the non-observation of horizontal showers [18, 19] by the Fly's Eye collaboration [20] and the Akeno Giant Air Shower Array (AGASA) collaboration 21, respectively. These constraints turn out to be competitive with other currently available constraints on TeV-scale gravity which are mainly based on interactions associated with Kaluza-Klein gravitons, according to which a fundamental Planck scale as low as $M_{D}=\mathcal{O}(1) \mathrm{TeV}$ is still allowed for $\delta \geq 4$ flat or $\delta \geq 1$ warped extra dimensions $[22]^{1}$.

In this Letter, we want to consider the sensitivity of neutrino telescopes such as AMANDA/IceCube [23], ANTARES [24, Baikal [25], NESTOR [26], and RICE [27] to black hole production in the scattering of ultrahigh energy cosmic neutrinos on nucleons in ice or water, considerably extending a first exploratory study [28]. After briefly reviewing the phenomenological model of black hole production adopted, we calculate the rate expectation for events contained in the fiducial volume of a neutrino telescope. We then extend our calculations to rates from throughgoing muons. Finally, we discuss the achievable sensitivity for detection of black hole production and compare it with that from air-shower facilities and the LHC.

2. Our phenomenological model of black hole production and decay will be based on the following working hypothesis which summarizes the current understanding of the production and decay of black holes in TeV-scale gravity scenarios [4, 15, 6, 8, 9, 10, 11].

It is assumed that at trans-Planckian parton-parton cm energies squared, $\hat{s} \gg M_{D}^{2}$, and at small parton-parton impact parameters, $b \ll r_{S}\left(M_{\mathrm{bh}}=\sqrt{\hat{s}}\right)$, i. e. at impact parameters much smaller than the Schwarzschild radius $r_{S}$ of a $(4+\delta)$-dimensional black hole with mass $M_{\mathrm{bh}}=\sqrt{\hat{s}}[29]$,

$$
r_{S}=\frac{1}{M_{D}}\left[\frac{M_{\mathrm{bh}}}{M_{D}}\left(\frac{2^{\delta} \pi^{\frac{\delta-3}{2}} \Gamma\left(\frac{3+\delta}{2}\right)}{2+\delta}\right)\right]^{\frac{1}{1+\delta}},
$$

\footnotetext{
${ }^{1}$ For an exhaustive list of references in this context, see also Ref. [16].

${ }^{2}$ We define $M_{D}$ as in Ref. 30. Equation (10) is valid as long as $r_{S} \ll R_{c}$, with $R_{c}$ being the compactification or curvature radii in the flat or warped scenario, respectively.
} 
a black hole forms with a cross section

$$
\hat{\sigma}(i j \rightarrow \mathrm{bh}) \equiv \hat{\sigma}_{i j}^{\mathrm{bh}}(\hat{s}) \approx \pi r_{S}^{2}\left(M_{\mathrm{bh}}=\sqrt{\hat{s}}\right) \theta\left(\sqrt{\hat{s}}-M_{\mathrm{bh}}^{\min }\right)
$$

Here, $M_{\mathrm{bh}}^{\min } \gg M_{D}$ parametrizes the cm energy above which the semiclassical reasoning mentioned above is assumed to be valid. The contribution of black hole production to the neutrino-nucleon cross section is then obtained as the convolution

$$
\sigma_{\nu N}^{\mathrm{bh}}(s)=\sum_{i} \int_{0}^{1} \mathrm{~d} x f_{i}(x, \mu) \hat{\sigma}_{\nu i}^{\mathrm{bh}}(x s) .
$$

Here, $s=2 m_{N} E_{\nu}$, with $m_{N}=\left(m_{p}+m_{n}\right) / 2$ denoting an isoscalar nucleon mass and $E_{\nu}$ the neutrino energy in the laboratory frame, denotes the neutrino-nucleon $\mathrm{cm}$ energy squared. The sum extends over all partons in the nucleon, with parton distribution functions $f_{i}(x, \mu)$ and factorization scale $\mu$. As in Ref. [16, we have used in the calculation of the cross section (31) various sets of parton distributions as they are implemented in the parton distribution library PDFLIB 31. In the following we display only the results obtained with the CTEQ3D 32 parton distributions with $\mu=\min (\sqrt{\hat{s}}, 10 \mathrm{TeV})$, keeping in mind that uncertainties associated with different parton distribution sets are in the $\mathcal{O}(20) \%$ range, whereas a choice of the other natural factorization scale $\mu=r_{S}^{-1}$ [8, 13] decreases the predicted rates by a factor of $\mathcal{O}(2)$.

It is fair to say that there remain nevertheless substantial uncertainties in the size of the cross section. As noted in Ref. 33, the semiclassical production of black holes resembles largely the problem of baryon and lepton number violating processes ("sphaleron 34 production") in multi$\mathrm{TeV}\left(\sqrt{\hat{s}} \gg m_{W} / \alpha_{W}\right)$ particle collisions in the standard electroweak theory [35, 36, 37] and more generally the problem of multi-particle production in weakly coupled theories [38, which is not yet completely understood [39]. There might be an additional exponential suppression factor rendering semiclassical sphaleron, multi-particle or black hole production unobservable in the $\mathrm{TeV}$ range [33] (see, however, Ref. [10]). Nevertheless, in this Letter we shall confine ourselves to the simple geometric cross section estimate (3) and postpone an inclusion of a possible exponential suppression factor to a longer write-up [40].

After production, the black holes decay primarily via Hawking radiation [41, corresponding to a temperature

$$
T_{\mathrm{H}}=\frac{\delta+1}{4 \pi r_{S}}
$$

into a large number [9],

$$
\langle n\rangle \approx \frac{1}{2} \frac{M_{\mathrm{bh}}}{T_{\mathrm{H}}},
$$

of hard quanta (cf. Fig. 11), with energies approaching several hundreds of GeV in the cm system. We shall assume that the average multiplicity (5) refers to Standard Model particles [5] - an assumption which is still under debate [42] - in a "flavor"-democratic fashion.

3. Let us consider now the rate of black hole events taking place within the fiducial volume of a neutrino telescope. The number of black hole events initiated by neutrino-nucleon scattering 


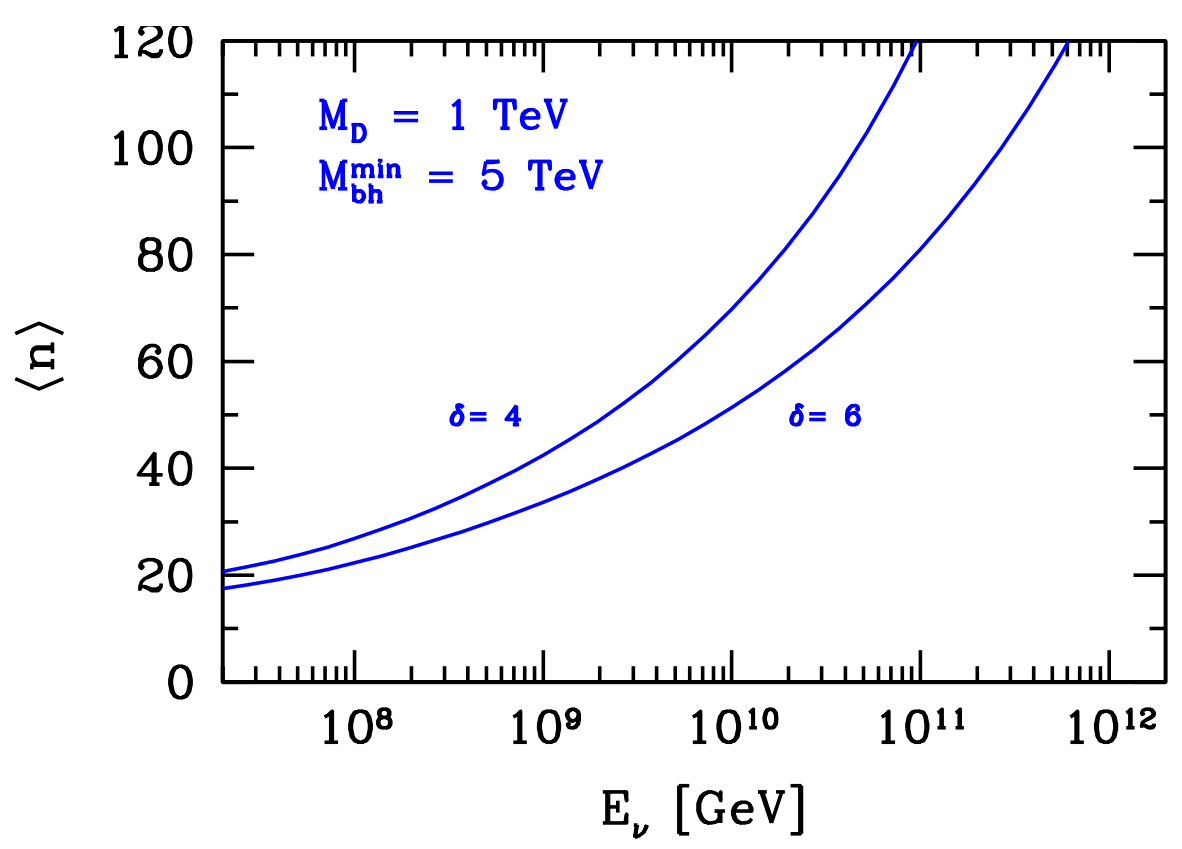

Figure 1: The average number $\langle n\rangle$ of Standard Model particles (5), into which a black hole produced in neutrino-nucleon scattering decays, as a function of the incident neutrino energy in the laboratory frame, $E_{\nu}$.

occurring per unit time $t$ and solid angle $\Omega$ with energy larger than a threshold energy $E_{\text {th }}$ inside a subsurface detector volume $V$ is (see e.g. Ref. [36])

$$
\frac{\mathrm{d}^{2} N_{\mathrm{casc}}^{\mathrm{bh}}}{\mathrm{d} t \mathrm{~d} \Omega}=\frac{\rho V}{m_{N}} \int_{E_{\mathrm{th}}}^{\infty} \mathrm{d} E_{\nu} F_{\nu}\left(E_{\nu}\right) \sigma_{\nu N}^{\mathrm{bh}}\left(E_{\nu}\right) \exp \left[-\sigma_{\nu N}^{\mathrm{tot}}\left(E_{\nu}\right) X(\theta) / m_{N}\right],
$$

where $\rho$ is the density of the material in which the neutrino interaction occurs, $F_{\nu}=\sum_{i}\left(F_{\nu_{i}}+F_{\bar{\nu}_{i}}\right)$ is the sum of the differential diffuse neutrino fluxes, $\sigma_{\nu N}^{\text {tot }}$ denotes the total interaction cross section as a sum of the usual Standard Model charged current cross section $\sigma_{\nu N}^{\mathrm{cc}}$ and the black hole production cross section (3),

$$
\sigma_{\nu N}^{\mathrm{tot}}=\sigma_{\nu N}^{\mathrm{cc}}+\sigma_{\nu N}^{\mathrm{bh}}
$$

and, finally, $X(\theta)$ is the column density of material between the detector and the upper atmosphere, as a function of the zenith angle $\theta$. It can be approximated by [36]

$$
X(\theta)=\rho\left[\sqrt{\left(R_{\oplus}-D\right)^{2} \cos ^{2} \theta+2 D R_{\oplus}-D^{2}}-\left(R_{\oplus}-D\right) \cos \theta\right],
$$

where $R_{\oplus}$ denotes the radius of the earth and $D$ is the vertical depth of the detector.

As in Ref. [16], we shall exploit both conservative lower and upper limits [43, 44] on the presently unknown differential flux $F_{\nu}$ of ultrahigh energy cosmic neutrinos (for recent reviews, see Ref. 45]) entering into event rates at neutrino telescopes such as Eq. (6). More or less guaranteed, and therefore comprising a reasonable lower bound, are the so-called cosmogenic neutrinos which are produced when ultrahigh energy cosmic protons inelastically scatter off the cosmic microwave 


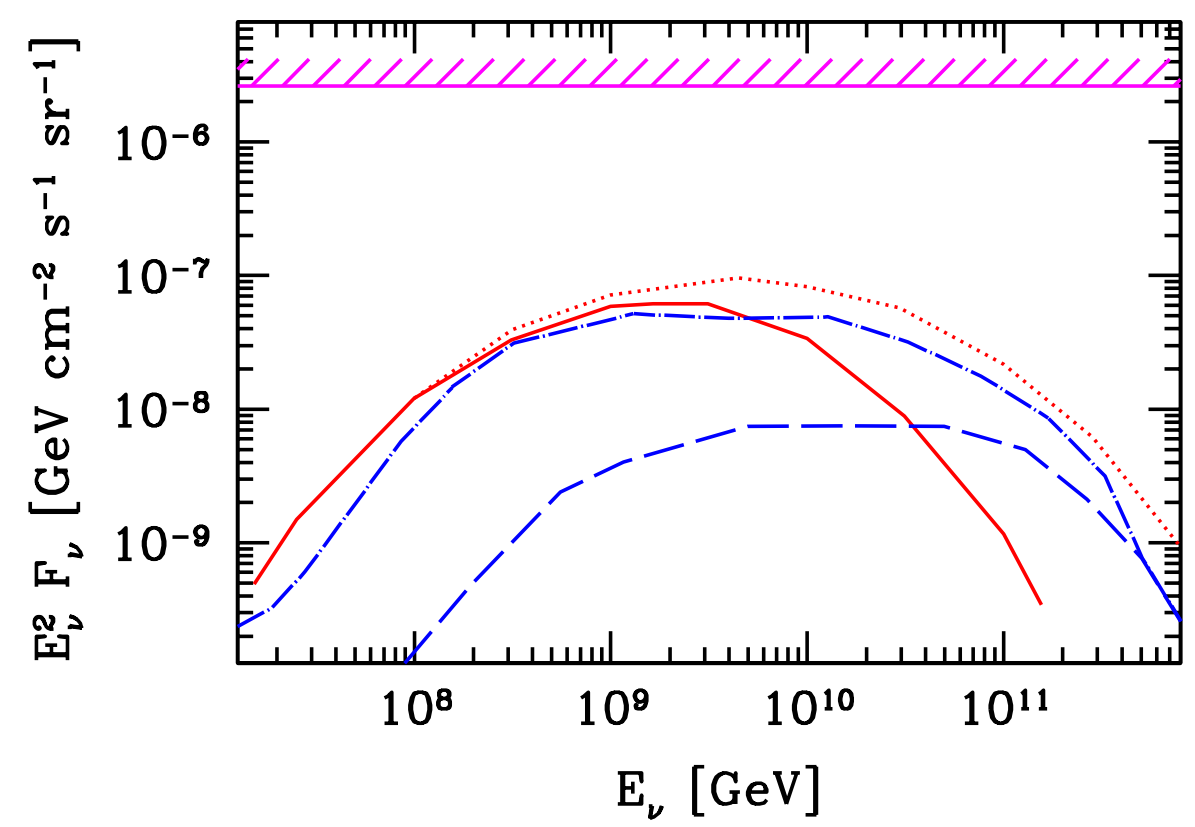

Figure 2: Predictions of the cosmogenic neutrino flux, $F_{\nu}=\sum_{i}\left[F_{\nu_{i}}+F_{\bar{\nu}_{i}}\right]$. Long-dashed (longdashed-dotted) line: Flux from Ref. [48 for cosmological evolution parameters $m=2, z_{\max }=2$ $\left(m=4, z_{\max }=4\right)$. Solid (dotted) line: Flux from Ref. 49], assuming a maximum energy of $E_{\max }=3 \cdot 10^{20(21)} \mathrm{eV}$ for the ultrahigh energy cosmic rays. Shaded: Theoretical upper limit of the neutrino flux from "hidden" astrophysical sources that are non-transparent to ultrahigh energy nucleons 44 .

background radiation [46] in processes such as $p \gamma \rightarrow \Delta \rightarrow n \pi^{+}$, where the produced pion subsequently decays [47]. Recent estimates of these fluxes can be found in Refs. [48, 49, 50, 51, some of which are shown in Fig. 2, The upper limit from "hidden" hadronic astrophysical sources, 3 , i. e. from those sources from which only photons and neutrinos can escape, is much larger [44] and also shown in Fig. 2

The projected reach in the black hole production parameter space for $\delta=6$ extra dimensions for contained events in an under-ice detector corresponding to the IceCube proposal 23 ] $(2 \mathrm{~km}$ depth, fiducial volume $1 \mathrm{~km}^{3}$ ) is shown in Fig. 3, for two of the discussed ultrahigh energy cosmic neutrino fluxes. Table 1 summarizes the corresponding expected Standard Model background rates for contained events due to charged current interactions. Taking into account the small effective volume, $V \approx 0.001 \div 0.01 \mathrm{~km}^{3}$, of the currently operating AMANDA and Baikal neutrino telescopes, it seems that, on the basis of contained events, these facilities cannot compete with the already established limits from air shower observatories such as Fly's Eye and AGASA, and the projected ones from Auger [12, 16, 17]. Even IceCube does not seem to be really competitive, as far as the contained events are concerned: According to the time schedule of IceCube, the construction starts in 2004 and the final effective volume, $V \approx 1 \mathrm{~km}^{3}$, is reached only after the

\footnotetext{
${ }^{3}$ For an early determination of such an upper limit, see Ref. [52. Fluxes of this size are required in the context of the Z-burst scenario [53] for the highest energy cosmic rays.
} 

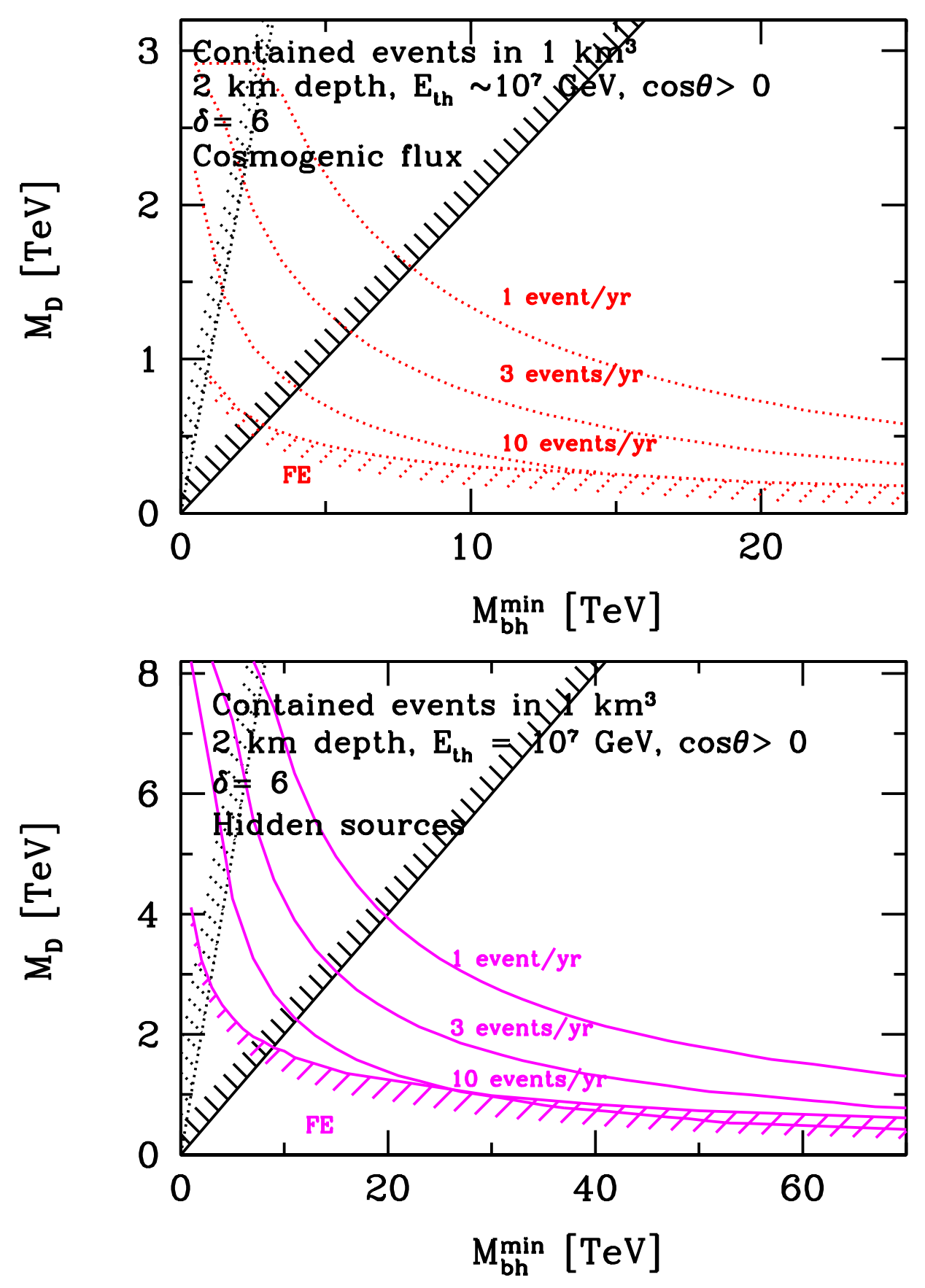

Figure 3: Reach in the black hole production parameters for $\delta=6$ extra dimensions, for contained events in an under-ice detector at a depth of $2 \mathrm{~km}$ and with an $1 \mathrm{~km}^{3}$ ficudial volume $(1 \mathrm{yr}=$ $\left.3.16 \cdot 10^{7} \mathrm{~s}\right)$. The shaded solid lines labeled "FE" indicates the constraint 16] arising from the non-observation of horizontal showers by the Fly's Eye collaboration [18. The shaded dotted, $M_{D}=M_{\mathrm{bh}}^{\min }$, and shaded solid, $M_{D}=(1 / 5) M_{\mathrm{bh}}^{\min }$, lines give a rough indication of the boundary of applicability of the semiclassical picture [8]. Top: Exploiting the cosmogenic neutrino flux from Ref. 49] (cf. Fig. 2). Bottom: Exploiting the upper limit on the neutrino flux from "hidden" hadronic astrophysical sources from Ref. [4] (cf. Fig. 22). 


\begin{tabular}{|c||c|c|c|c|}
\hline \multicolumn{1}{|c||}{} & \multicolumn{2}{c|}{ cosmogenic neutrinos } & \multicolumn{2}{c|}{ hidden sources } \\
\hline$E_{\mathrm{th}}$ & contained & through-going & contained & through-going \\
\hline $10^{7} \mathrm{GeV}$ & $0.47\left(\mathrm{yr} \mathrm{km}^{3}\right)^{-1}$ & $0.85\left(\mathrm{yr} \mathrm{km}^{2}\right)^{-1}$ & $116\left(\mathrm{yr} \mathrm{km}^{3}\right)^{-1}$ & $92\left(\mathrm{yr} \mathrm{km}^{2}\right)^{-1}$ \\
\hline
\end{tabular}

Table 1: Event rates (1 yr $\left.=3.16 \cdot 10^{7} \mathrm{~s}\right)$ of contained reactions and through-going muons, respectively, for Standard Model like cross sections for the case of the cosmogenic neutrino flux from Ref. [49], assuming a maximum energy of $E_{\max }=3 \cdot 10^{21} \mathrm{eV}$ for the ultrahigh energy cosmic rays, and the case of the upper limit on the neutrino flux from "hidden" hadronic astrophysical sources from Ref. [4], respectively. An under-ice detector at a depth of $2 \mathrm{~km}$ has been assumed. For the contained events, we have considered, as in Eq. (77), charged current reactions from all flavors, but have neglected neutral current reactions, because the fraction of visible energy, $\langle y\rangle \approx 0.2$, as well as the cross section, is lower for the latter [54]. For the through-going events, we have considered charged current $\nu_{\mu} N$ and $\bar{\nu}_{\mu} N$ interactions and an equipartion of neutrino flavors in the neutrino flux.

LHC starts operating and Auger has taken data for already a few years. For the under-water/ice neutrino telescopes considered so far, the best perspective for detection of black hole events on the basis of the contained events might thus be assigned to RICE [27], a currently operating radio-Cherenkov telescope with an effective volume of about $1 \mathrm{~km}^{3}$ for $10^{8} \mathrm{GeV}$ electromagnetic cascades 55 .

4. The ability to detect muons from distant neutrino reactions increases an under-ice/water detector's effective neutrino target volume dramatically and is the premise upon which such detectors can act as neutrino telescopes. Let us therefore, in addition to the rate (6) of fully contained black hole events, estimate the rate of through-going muons at a neutrino telescope. We define through-going muon events to be events with a vertex located outside of the detector and which then pass through the detector. The expected rate for such events is then proportional to the area of the detector. The muon rate above some threshold $E_{\text {th }}$ per unit area $A$ and solid angle $\Omega$ can be estimated by

$$
\begin{aligned}
\frac{\mathrm{d}^{3} N_{\mu}^{\mathrm{bh}}}{\mathrm{d} A \mathrm{~d} t \mathrm{~d} \Omega}= & \sum_{k=1}^{\infty} \frac{1}{m_{N}} \int_{E_{\mathrm{th}}}^{\infty} \mathrm{d} E_{\nu} F_{\nu}\left(E_{\nu}\right) \int \mathrm{d} n_{\mu} \frac{\mathrm{d} \sigma_{\nu N}^{\mathrm{bh}}}{\mathrm{d} n_{\mu}} \frac{n_{\mu}^{k}}{k !} \mathrm{e}^{-n_{\mu}} \times \\
& \times \int_{0}^{X(\theta)} \mathrm{d} X^{\prime} \exp \left[-\sigma_{\nu N}^{\mathrm{tot}}\left(E_{\nu}\right)\left(X(\theta)-X^{\prime}\right) / m_{N}\right]\left(1-\left(1-\tilde{p}_{\mu}^{\mathrm{bh}}\left(E_{\nu}, E_{\mathrm{th}}, X^{\prime}\right)\right)^{k}\right) .
\end{aligned}
$$

In words, the rate of through-going muon events from a black hole reaction is obtained by summing over different muon multiplicities $k$, weighted with the Poisson probability of mean $n_{\mu}$ that $k$ muons emerge from black hole decay and that at least one can be observed in the detector. The quantity $\tilde{p}_{\mu}^{\text {bh }}$, which may be interpreted as the detection probability of a "typical" muon produced in a black hole event, is defined as (cf. also Ref. [36])

$$
\tilde{p}_{\mu}^{\mathrm{bh}}\left(E_{\nu}, E_{\mathrm{th}}, X^{\prime}\right)=\int_{E_{\mathrm{th}}}^{E_{\nu}} \mathrm{d} E_{\mu} \frac{1}{n_{\mu}^{\mathrm{bh}}} \frac{\mathrm{d} n_{\mu}^{\mathrm{bh}}}{\mathrm{d} E_{\mu}} p_{\mu}\left(E_{\mu}, E_{\mathrm{th}}, X^{\prime}\right)
$$

with $p_{\mu}\left(E_{\mu}, E_{\mathrm{th}}, X^{\prime}\right)$ denoting the probability that a muon of definite energy $E_{\mu}$ traverses an amount $X^{\prime}$ of material with $E_{\mu}>E_{\mathrm{th}}$. 

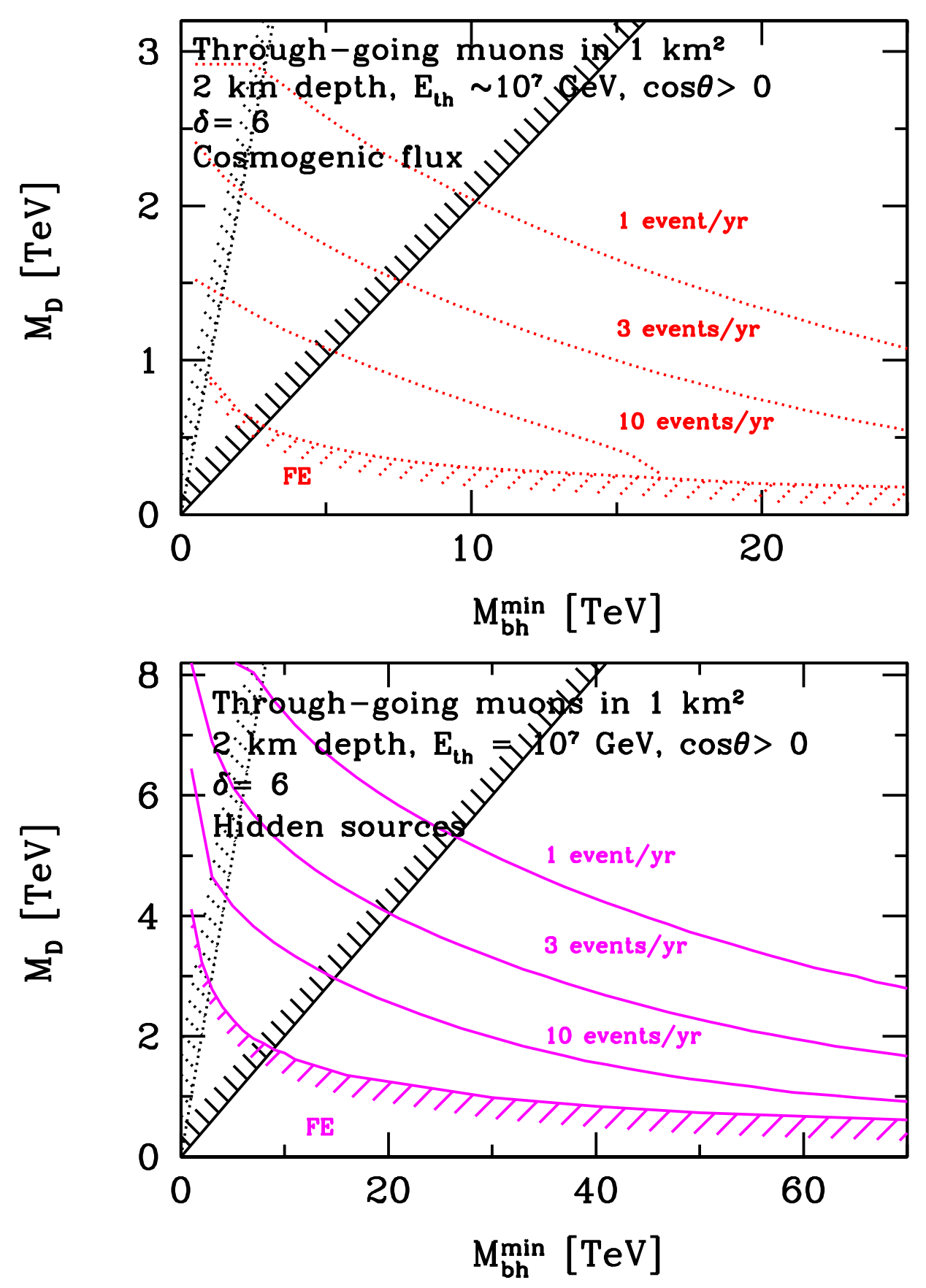

Figure 4: Reach in the black hole production parameters for $\delta=6$ extra dimensions, for throughgoing muons in an under-ice detector at a depth of $2 \mathrm{~km}$ and with an $1 \mathrm{~km}^{2}$ effective area $\left(1 \mathrm{yr}=3.16 \cdot 10^{7} \mathrm{~s}\right)$. The shaded solid lines labeled "FE" indicates the constraint [16] arising from the non-observation of horizontal showers by the Fly's Eye collaboration [18]. The shaded dotted, $M_{D}=M_{\mathrm{bh}}^{\min }$, and shaded solid, $M_{D}=(1 / 5) M_{\mathrm{bh}}^{\min }$, lines give a rough indication of the boundary of applicability of the semiclassical picture [8]. Top: Exploiting the cosmogenic neutrino flux from Ref. 49] (cf. Fig. 2). Bottom: Exploiting the upper limit on the neutrino flux from "hidden" hadronic astrophysical sources from Ref. [4] (cf. Fig. 22). 
If one neglects the stochastic effects of range straggling which may become important for muon energies close to the detection threshold, i.e. within the approximation of a deterministic energyrange relationship, one has the step function

$$
p_{\mu}\left(E_{\mu}, E_{\mathrm{th}}, X^{\prime}\right)=\theta\left(E_{\mu}-E_{\mu}^{\prime}\left(E_{\mathrm{th}}, X^{\prime}\right)\right)
$$

where $E_{\mu}^{\prime}$ is the solution of

$$
X^{\prime}=\int_{E_{\mathrm{th}}}^{E_{\mu}^{\prime}} \frac{\mathrm{d} E}{a(E)+b(E) E} .
$$

The ionization and radiation loss parameters, $a(E)$ and $b(E)$, vary only slowly with energy and take the asymptotic values $a \approx 3 \cdot 10^{-3} \mathrm{GeV} \mathrm{cm} / \mathrm{g}$ and $b \approx 4 \cdot 10^{-6} \mathrm{~cm}^{2} / \mathrm{g}$ [56]. Choosing these constant values, we get for the average muon range,

$$
X^{\prime} \approx \frac{1}{b} \ln \frac{a+b E_{\mu}^{\prime}}{a+b E_{\mathrm{th}}} \quad \Leftrightarrow \quad E_{\mu}^{\prime}\left(E_{\mathrm{th}}, X^{\prime}\right) \approx\left[E_{\mathrm{th}}+\left(1-\mathrm{e}^{-b X^{\prime}}\right) \frac{a}{b}\right] \mathrm{e}^{b X^{\prime}} .
$$

Since for our considerations $E_{\mathrm{th}} \sim 10^{7} \mathrm{GeV}$, we can safely neglect the contribution proportional to $a$ in the following.

For the laboratory energy distribution of the muons produced in a black hole event, entering the probability (10), we have taken for simplicity (cf. also Ref. [36])

$$
\frac{1}{n_{\mu}^{\mathrm{bh}}} \frac{\mathrm{d} n_{\mu}^{\mathrm{bh}}}{\mathrm{d} E_{\mu}}=\frac{\langle n\rangle}{2 E_{\nu}} \theta\left(\frac{2 E_{\nu}}{\langle n\rangle}-E_{\mu}\right)
$$

with $\langle n\rangle$ being the average number (5) of particles produced in black hole decay (cf. Fig. 11). The distribution (14) is obtained by boosting a delta-function energy spectrum,

$$
\frac{\mathrm{d} \sigma_{\nu N}^{\mathrm{bh}}}{\mathrm{d} E_{\mu}^{\mathrm{cm}}} \propto \delta\left(E_{\mu}^{\mathrm{cm}}-M_{\mathrm{bh}} /\langle n\rangle\right)
$$

in the cm frame of the black hole, with isotropic direction of the decay particles, with a Lorentz factor $\gamma=E_{\nu} / M_{\mathrm{bh}}$ to the laboratory frame.

Finally, we have exploited the ansatz (similar to Eq.(15) ),

$$
\frac{d \sigma_{\nu N}^{\mathrm{bh}}}{\mathrm{d} n_{\mu}}=\sigma_{\nu N}^{\mathrm{bh}} \delta\left(n_{\mu}-\left\langle n_{\mu}\right\rangle\right), \quad \text { with }\left\langle n_{\mu}\right\rangle \approx\langle n\rangle / 30
$$

to perform also the integration over the multiplicity $n_{\mu}$ in our rate estimate (9). The above relation for $\left\langle n_{\mu}\right\rangle$ is based on the assumption that a black hole does not discriminate between Standard Model particles in its evaporation spectrum [8].

The projected reach in the black hole production parameter space for $\delta=6$ extra dimensions for through-going muons in an under-ice detector at a depth of $2 \mathrm{~km}$ and with an effective area

\footnotetext{
${ }^{4}$ Alternatively, one may use a boosted thermal distribution, $\sim \int_{E_{\mu} / 2 \gamma}^{\infty} \mathrm{d} E\left(E / T_{\mathrm{H}}\right)^{2} /\left(e^{E / T_{\mathrm{H}}}+1\right)$, which, however, needs an additional numerical integration.
} 
of $1 \mathrm{~km}^{2}$ is shown in Fig. 4, for two of the discussed ultrahigh energy cosmic neutrino fluxes. The corresponding expected Standard Model background rates for through-going muons due to charged current interactions is summarized in Table 1 (see also Ref. [57] for related calculations). An additional source of background which is not related to an astrophysical neutrino flux is the muon flux arising from charm production by cosmic rays interacting in the atmosphere. Using two available models [58, 59] we have estimated a background of $0.33 \div 1.5$ through-going muon events from atmospheric charm production per year. To further reduce this background, one may introduce a cut in the zenith angle, e.g. $\theta>60^{\circ}$.

5. We have considered the sensitivity of neutrino telescopes to black hole production in the context of extra dimension scenarios with $\mathrm{TeV}$-scale gravity and compared it to the one of air shower facilities and the LHC. For concreteness, we have assumed a detector with $1 \mathrm{~km}^{2}$ area and $1 \mathrm{~km}^{3}$ volume, respectively, located at a vertical depth of $2 \mathrm{~km}$ in ice.

We have seen that the effective volumina for contained events, $V \approx 0.001 \div 0.01 \mathrm{~km}^{3}$, available from the currently operating AMANDA and Baikal detectors are too small to yield a large enough rate to be competitive with the already existing constraints on black hole production from Fly's Eye and AGASA and with the projected ones from Auger [12, 16, 17. The planned IceCube detector with a fiducial volume of about $1 \mathrm{~km}^{3}$ would have an interesting perspective. However, with the start of construction of IceCube in 2004 and full completion only after the physics start at the LHC, the discovery potential for black hole production is strongly reduced. Instead, the radio-Cherenkov telescope RICE [27, with an effective volume of about 0.1 (1) $\mathrm{km}^{3}$ for $10^{7(8)} \mathrm{GeV}$ electromagnetic cascades [55, could set sensible constraints using already available data.

The prospects for a search for black hole signatures using through-going muons are also good. For a detection area of $1 \mathrm{~km}^{2}$ we have shown that in case the neutrino flux is just on the level of the cosmogenic one, only a few $(\lesssim 1)$ contained or through-going background events from Standard Model charged current interactions are expected per year. The background of prompt muons from atmospheric charm was estimated to be of similar magnitude and can be further reduced by application of a cut on the zenith angle. It was shown in Ref. [60], that AMANDA achieves an effective area of about $0.3 \mathrm{~km}^{2}$ for down-going muons above an energy of $10^{7} \mathrm{GeV}$ and that suppression of the atmospheric muon background is possible. With 5 years of AMANDA data available and an assumed duty-factor of $2 / 3$ this leads to an exposure of about $1 \mathrm{~km}^{2} \cdot \mathrm{yr}$. Therefore, strong constraints on the black hole production parameters are expected from AMANDA if the currently available data show no through-going muon 5 above $10^{7} \mathrm{GeV}$. In this case, the sensitivity of AMANDA - approximately the 3 events/yr contour in Fig. 4 (top) for a $90 \%$ confidence level limit - compares favorably with the one of Fly's Eye, AGASA, and Auger [12, 16, 17. And even at the kinematic limit of the $\mathrm{LHC}_{\mathrm{bh}}^{\mathrm{min}} \approx 10 \mathrm{TeV}$ (cf. Ref. [16]), one is still left with a sensitivity on $M_{D}$ of about $1.3 \mathrm{TeV}$.

In the case that the ultrahigh energy cosmic neutrino flux considerably exceeds the cosmogenic one, discrimination between Standard Model background and black hole events becomes crucial. This might not be achieved by observation of through-going events alone, but with the additional information of contained events. For example, the spectral information from contained

\footnotetext{
${ }^{5}$ It is noteworthy that in this case AMANDA can put also strong constraints on sphaleron production parameters [36], since the phenomenology of black hole and sphaleron production are quite similar.
} 
and through-going events should be remarkably different, as the through-going muons from black hole decays are produced with considerably lower energy. Another discriminator may be based on the different hadronic components of Standard Model processes and black hole events. In a typical Standard Model charged current interaction one has an average hadronic energy fraction of $\langle y\rangle \approx 0.2$, whereas black hole events have typically a large hadronic component $(5: 1)[8]$ corresponding to a $\langle y\rangle \approx 0.8$. In particular radio-Cherenkov detectors such as RICE have the potential to discriminate between electromagnetic and hadronic showers by measuring the characteristically different angular radiation patterns [61].

To summarize, we have shown that large scale under-water/ice neutrino detectors have a substantial potential to discover or rule out black hole production which is comparable to that of air shower facilities. In the optimistic case that a neutrino flux significantly higher than the cosmogenic one is realized in nature, one even has discovery potential for black holes at neutrino telescopes beyond the reach of LHC.

\section{Acknowledgements}

We thank C. Spiering for fruitful discussions.

\section{References}

[1] G. 't Hooft, Phys. Lett. B 198 (1987) 61; Nucl. Phys. B 304 (1988) 867; D. Amati, M. Ciafaloni and G. Veneziano, Phys. Lett. B 197 (1987) 81; Int. J. Mod. Phys. A 3 (1988) 1615; Phys. Lett. B 216 (1989) 41; Nucl. Phys. B 347 (1990) 550; Phys. Lett. B 289 (1992) 87; Nucl. Phys. B 403 (1993) 707; M. Fabbrichesi, R. Pettorino, G. Veneziano and G. A. Vilkovisky, Nucl. Phys. B 419 (1994) 147; I. Y. Aref'eva, K. S. Viswanathan and I. V. Volovich, Nucl. Phys. B 452 (1995) 346 [Erratum-ibid. B 462 (1995) 613].

[2] N. Arkani-Hamed, S. Dimopoulos and G. R. Dvali, Phys. Lett. B 429 (1998) 263; I. Antoniadis, N. Arkani-Hamed, S. Dimopoulos and G. R. Dvali, Phys. Lett. B 436 (1998) 257; N. Arkani-Hamed, S. Dimopoulos and G. R. Dvali, Phys. Rev. D 59 (1999) 086004.

[3] L. J. Randall and R. Sundrum, Phys. Rev. Lett. 83 (1999) 3370.

[4] P. C. Argyres, S. Dimopoulos and J. March-Russell, Phys. Lett. B 441 (1998) 96; T. Banks and W. Fischler, hep-th/9906038; I. Y. Aref'eva, hep-th/9910269.

[5] R. Emparan, G. T. Horowitz and R. C. Myers, Phys. Rev. Lett. 85 (2000) 499.

[6] S. B. Giddings and E. Katz, hep-th/0009176; R. Emparan, Phys. Rev. D 64 (2001) 024025.

[7] L. R. Evans, CERN-OPEN-2001-027, presented at: 18th International Conference on High Energy Accelerators, Tsukuba, Japan, 26 - 30 Mar 2001.

[8] S. B. Giddings and S. Thomas, hep-ph/0106219. 
[9] S. Dimopoulos and G. Landsberg, Phys. Rev. Lett. 87 (2001) 161602.

[10] S. Dimopoulos and R. Emparan, hep-ph/0108060.

[11] S. Hossenfelder, S. Hofmann, M. Bleicher and H. Stöcker, hep-ph/0109085 S. B. Giddings, hep-ph/0110127; K. Cheung, hep-ph/0110163 S. C. Park and H. S. Song, hep-ph/0111069; T. G. Rizzo, hep-ph/0111230; G. Landsberg, hep-ph/0112061; G. F. Giudice, R. Rattazzi and J. D. Wells, hep-ph/0112161; M. Bleicher, S. Hofmann, S. Hossenfelder and H. Stöcker, hep-ph/0112186, D. M. Eardley and S. B. Giddings, gr-qc/0201034.

[12] J. L. Feng and A. D. Shapere, Phys. Rev. Lett. 88 (2002) 021303.

[13] R. Emparan, M. Masip and R. Rattazzi, hep-ph/0109287.

[14] L. Anchordoqui and H. Goldberg, hep-ph/0109242.

[15] D. Zavrtanik [AUGER Collaboration], Nucl. Phys. Proc. Suppl. 85 (2000) 324.

[16] A. Ringwald and H. Tu, Phys. Lett. B 525 (2002) 135.

[17] L. A. Anchordoqui, J. L. Feng, H. Goldberg and A. D. Shapere, hep-ph/0112247.

[18] R. M. Baltrusaitis et al., Phys. Rev. D 31 (1985) 2192.

[19] S. Yoshida et al. [AGASA Collaboration], in: Proc. 27th International Cosmic Ray Conference, Hamburg, Germany, 2001, Vol. 3, pp. 1142-1145.

[20] R. M. Baltrusaitis et al., Nucl. Instrum. Meth. A 240 (1985) 410.

[21] N. Chiba et al., Nucl. Instrum. Meth. A 311 (1992) 338.

[22] M. E. Peskin, hep-ph/0002041; T. Abe et al. [American Linear Collider Working Group Collaboration], hep-ex/0106057; C. Pagliarone, hep-ex/0111063.

[23] F. Halzen et al. [AMANDA Collaboration], in: B.L. Dingus, D.B. Kieda, M.H. Salamon (Eds.), 26th International Cosmic Ray Conference (ICRC 99), Salt Lake City, UT, 17-25 Aug 1999, Melville, AIP, 2000, pp. 428-431.

[24] T. Montaruli [ANTARES Collaboration], hep-ex/0201009.

[25] V. Balkanov et al. [Baikal Collaboration], astro-ph/0112446

[26] P. K. Grieder [NESTOR Collaboration], Nucl. Phys. Proc. Suppl. 97 (2001) 105.

[27] I. Kravchenko et al. [RICE Collaboration], astro-ph/0112372

[28] Y. Uehara, hep-ph/0110382.

[29] R. C. Myers and M. J. Perry, Annals Phys. 172 (1986) 304. 
[30] G. F. Giudice, R. Rattazzi and J. D. Wells, Nucl. Phys. B 544 (1999) 3 and erratum to appear hep-ph/9811291 v2].

[31] H. Plothow-Besch, Comput. Phys. Commun. 75 (1993) 396; Int. J. Mod. Phys. A 10 (1995) 2901; http://consult.cern.ch/writeups/pdflib/main.ps

[32] H. L. Lai et al., Phys. Rev. D 51 (1995) 4763.

[33] M. B. Voloshin, Phys. Lett. B 518 (2001) 137; Phys. Lett. B 524 (2002) 376.

[34] F. R. Klinkhamer and N. S. Manton, Phys. Rev. D 30 (1984) 2212.

[35] A. Ringwald, Nucl. Phys. B 330 (1990) 1; O. Espinosa, Nucl. Phys. B 343 (1990) 310; L. D. McLerran, A. I. Vainshtein and M. B. Voloshin, Phys. Rev. D 42 (1990) 171; A. Ringwald and C. Wetterich, Nucl. Phys. B 353 (1991) 303; A. Ringwald, F. Schrempp and C. Wetterich, Nucl. Phys. B 365 (1991) 3.

[36] D. A. Morris and R. Rosenfeld, Phys. Rev. D 44 (1991) 3530; D. A. Morris and A. Ringwald, Astropart. Phys. 2 (1994) 43.

[37] M. J. Gibbs, A. Ringwald, B. R. Webber and J. T. Zadrozny, Z. Phys. C 66 (1995) 285.

[38] J. M. Cornwall, Phys. Lett. B 243 (1990) 271; H. Goldberg, Phys. Lett. B 246 (1990) 445.

[39] M. P. Mattis, Phys. Rept. 214 (1992) 159; P. G. Tinyakov, Int. J. Mod. Phys. A 8 (1993) 1823; M. B. Voloshin, hep-ph/9409344 V. A. Rubakov and M. E. Shaposhnikov, Usp. Fiz. Nauk 166 (1996) 493 [Phys. Usp. 39 (1996) 461].

[40] M. Kowalski, A. Ringwald and H. Tu, in preparation.

[41] S. W. Hawking, Nature 248 (1974) 30; Commun. Math. Phys. 43 (1975) 199.

[42] R. Casadio and B. Harms, hep-th/0110255.

[43] E. Waxman and J. N. Bahcall, Phys. Rev. D 59 (1999) 023002; J. N. Bahcall and E. Waxman, Phys. Rev. D 64 (2001) 023002.

[44] K. Mannheim, R. J. Protheroe and J. P. Rachen, Phys. Rev. D 63 (2001) 023003.

[45] R. J. Protheroe, Nucl. Phys. Proc. Suppl. 77 (1999) 465; R. Gandhi, Nucl. Phys. Proc. Suppl. 91 (2000) 453; J. G. Learned and K. Mannheim, Ann. Rev. Nucl. Part. Sci. 50 (2000) 679.

[46] K. Greisen, Phys. Rev. Lett. 16 (1966) 748; G. T. Zatsepin and V. A. Kuzmin, JETP Lett. 4 (1966) 78 [Pisma Zh. Eksp. Teor. Fiz. 4 (1966) 114].

[47] V. S. Berezinsky and G. T. Zatsepin, Phys. Lett. B 28 (1969) 423; V. S. Berezinsky and G. T. Zatsepin, Sov. J. Nucl. Phys. 11 (1970) 111 [Yad. Fiz. 11 (1970) 200].

[48] S. Yoshida and M. Teshima, Prog. Theor. Phys. 89 (1993) 833. 
[49] R. J. Protheroe and P. A. Johnson, Astropart. Phys. 4 (1996) 253 [Erratum-ibid. 5 (1996) 215].

[50] S. Yoshida, H. Dai, C. C. Jui and P. Sommers, Astrophys. J. 479 (1997) 547.

[51] R. Engel and T. Stanev, Phys. Rev. D 64 (2001) 093010.

[52] V. S. Berezinsky, in: J. G. Learned (Ed.), DUMAND Summer Workshops, Khabarovsk and Lake Baikal, 22-31 Aug 1979, Hawaii DUMAND Center, University of Hawaii, 1980, pp. $245-261$.

[53] D. Fargion, B. Mele and A. Salis, Astrophys. J. 517 (1999) 725; T. J. Weiler, Astropart. Phys. 11 (1999) 303; S. Yoshida, G. Sigl and S. J. Lee, Phys. Rev. Lett. 81 (1998) 5505; Z. Fodor, S. D. Katz and A. Ringwald, hep-ph/0105064; hep-ph/0105336 A. Ringwald, hep-ph/0111112 O. E. Kalashev, V. A. Kuzmin, D. V. Semikoz and G. Sigl, hep-ph/0112351.

[54] R. Gandhi, C. Quigg, M. H. Reno and I. Sarcevic, Astropart. Phys. 5 (1996) 81.

[55] D. Seckel et al. [RICE Collaboration], in: Proc. 27th International Cosmic Ray Conference, Hamburg, Germany, 2001, Vol. 3, pp. 1137-1140.

[56] D. E. Groom et al. [Particle Data Group Collaboration], Eur. Phys. J. C 15 (2000) 1.

[57] J. Alvarez-Muniz and F. Halzen, Phys. Rev. D 63 (2001) 037302.

[58] E. V. Bugaev, A. Misaki, V. A. Naumov, T. S. Sinegovskaya, S. I. Sinegovsky and N. Takahashi, Phys. Rev. D 58 (1998) 054001.

[59] G. Gelmini, P. Gondolo and G. Varieschi, Phys. Rev. D 61 (2000) 036005.

[60] S. Hundertmark et al. [AMANDA Collaboration], in: Proc. 27th International Cosmic Ray Conference, Hamburg, Germany, 2001, Vol. 3, pp. 1129-1132.

[61] J. Alvarez-Muniz, R. A. Vazquez and E. Zas, Phys. Rev. D 61 (2000) 023001. 